\title{
Image Reconstruction from Fan-Beam Projections without Back-Projection Weight in a 2-D Dynamic CT: Compensation of Time-Dependent Rotational, Uniform Scaling and Translational Deformations
}

\author{
A. V. Narasimhadhan ${ }^{1 *}$, Aman Sharma ${ }^{2}$ Dipen Mistry ${ }^{2}$ \\ ${ }^{1}$ National Institute of Technology Karnataka, Surathkal, India \\ ${ }^{2}$ National Institute of Technology Goa, Goa, India \\ Email: *dhan257gmail.com
}

Received September 5, 2013; revised October 5, 2013; accepted October 10, 2013

Copyright (C) 2013 A. V. Narasimhadhan et al. This is an open access article distributed under the Creative Commons Attribution License, which permits unrestricted use, distribution, and reproduction in any medium, provided the original work is properly cited.

\begin{abstract}
In a dynamic $\mathrm{CT}$, the acquired projections are corrupted due to strong dynamic nature of the object, for example: lungs, heart etc. In this paper, we present fan-beam reconstruction algorithm without position-dependent backprojection weight which compensates for the time-dependent translational, uniform scaling and rotational deformations occurring in the object of interest during the data acquisition process. We shall also compare the computational cost of the proposed reconstruction algorithm with the existing one which has position-dependent weight. To accomplish the objective listed above, we first formulate admissibility conditions on deformations that is required to exactly reconstruct the object from acquired sequential deformed projections and then derive the reconstruction algorithm to compensate the above listed deformations satisfying the admissibility conditions. For this, 2-D time-dependent deformation model is incorporated in the fan-beam FBP reconstruction algorithm with no backprojection weight, assuming the motion parameters being known. Finally the proposed reconstruction algorithm is evaluated with the motion corrupted projection data simulated on the computer.
\end{abstract}

Keywords: Fan-Beam Reconstruction Algorithm; Hilbert Filter; Virtual Acquisition Geometry; Equiangular Detector Geometry; Rotational; Uniform Scaling and Translational Deformation; Position Dependent Back-Projection Weight

\section{Introduction}

The object of interest being imaged may be dynamic in nature, hence it is very important to develop algorithms and techniques to compensate the motion artefacts in tomographic imaging. There are several techniques and algorithms developed to tackle the challenges posed by the dynamic nature of the object such as heart, lungs, etc. One straightforward approach would be to minimize or limit the data acquisition time using ultra fast scaffolds. But in practice, dedicated softwares must be implemented for good results. Different techniques have been developed for compensation of motion artefacts, namely, 1) Gating method by Kachelriess et al. and Flohr et al. $[1,2]$. 2) Integrating motion models in reconstruction

${ }^{*}$ Corresponding author. process using algebraic and analytical formulations [3-6]. 3) Reconstruction method for general type of motion with moderate amplitude $[7,8]$. 4) Method to correct the sinogram through simple motion models as global translations, magnifications or rotations [9]. 5) Reconstruction formula for compensation of translation and magnification [8].

Roux et al. [10] have proposed a new frame-work, wherein the real object, at a particular time, is mapped to a reference object at reference time through some transformation, thus giving rise to a virtual acquisition geometry. The key point to observe is that, the value of the Radon transform of the reference object, in the virtual geometry, at reference time is linked to the Radon transform of the transformed object, at a particular time. Thus giving rise to the image reconstruction formula from de- 
formed projections; involving parameters of the virtual acquisition geometry but the aforementioned reconstruction formula is convoyed with a position-dependent backprojection weight. Since large amount of data needs be processed in dynamic studies, the implementation of the FBP formula for dynamic CT proposed by Roux et al. [10] will consume more time than it's counterpart (static case). The solution to eliminate the position-dependent back-projection weight in the fan-beam FBP formula, proposed by Noo. et al. [11] inspired by [12] in a static 2-D CT was given in [13]. In this paper, we reduce the computational cost of the fan-beam FBP reconstruction formula for a 2-D dynamic CT proposed by Roux. et al., by eliminating the position-dependent back-projection weight, by choosing the weighting function similar to the one proposed in [13] for compensating time-dependent rotational, uniform scaling and translational deformations. By uniform scaling, we mean that the scaling transformation scales the object with the same magnitude along both the orthogonal reference co-ordinates. Thus for the above mentioned deformations, our formula will consume less time than the one proposed by Roux et al., due to the elimination of computationally expensive backprojection weight.

We follow the sequential approach listed below to address the issues raised in the paper. 1) Admissibility condition to reconstruct the object from the projections acquired during it's deformation (affine). 2) Reconstruction formula for a dynamic CT, compensating the time-dependent affine deformation. 3) Incorporating the techniques in the above formula to eliminate back-projection weight. 4) Simulating the idea presented in the paper.

\section{Theory}

Consider an object having strong dynamic nature and thus it deforms as a function of time during the data acquisition process. Let the object be denoted as $f_{t}$ at time $t$ and $f_{0}$ at time $t_{0}$, where $t_{0}$ is the reference time, where the object is assumed to be stationery. $\Gamma_{t}$ be the transformation that maps the real object at particular time $t$ to the reference time $t_{0}$ as shown in Figure 1. Therefore $\Gamma_{t}$ transforms the object from "real state" to "reference state".

$$
\begin{gathered}
f_{t}(\boldsymbol{x})=f_{0}(\Gamma(\boldsymbol{x})) \\
\Gamma_{t}(\boldsymbol{x})=A_{t}(\boldsymbol{x})+\boldsymbol{b}_{\boldsymbol{t}} \\
\text { where } A_{t}=\left[\begin{array}{ll}
a_{11}(t) & a_{12}(t) \\
a_{21}(t) & a_{22}(t)
\end{array}\right] \text { and } \boldsymbol{b}_{\boldsymbol{t}}=\left[\begin{array}{l}
b_{1}(t) \\
b_{2}(t)
\end{array}\right]
\end{gathered}
$$

Thus, each real X-ray path on the evolving object is associated with a virtual X-ray path on the reference object, giving rise to a virtual acquisition geometry. Since affine transformations preserve intersection, each

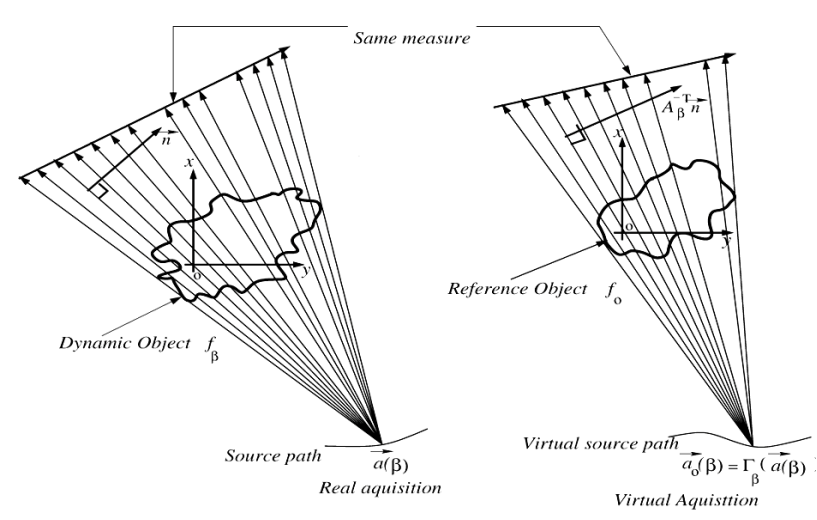

Figure 1. Fan-beam geometry: transformation of dynamic object projections to reference object projections (Courtesy: Roux et al. [10]).

fan beam projection is transformed into another fan beam projection.

In a fan beam geometry, the parameter $\beta$ uniquely determines one set of projection and also gives an understanding of the source location. Since $\beta$ is varied with respect to time $t$ for acquiring all the projections, therefore $t$ can be replaced with $\beta$ [10]. Mathematically,

$$
g_{\beta}(\beta, \alpha)=\int_{0}^{\infty}(a(\beta)+l \alpha) \mathrm{d} l
$$

$g_{\beta}(\beta, \alpha)$ be the dynamic fan-beam projection data collected with a circular scan trajectory given by $\boldsymbol{a}(\beta)=\left[\begin{array}{lll}R_{0} \cos \beta & R_{0} \sin \beta\end{array}\right]^{\mathrm{T}}$ for a dynamic object $f_{\beta}$ where $R_{0}$ is the radius of the source trajectory. $l \in \mathbb{R}^{+}$ and $\alpha \in \mathbf{S}^{1}$ where $\mathbf{S}^{1}$ is the set of unit vectors in $\mathbb{R}^{2}$.

\subsection{Admissibility Conditions on the Deformation for Fan-Beam Case}

Roux et al. have proposed a derivation of admissibility conditions based on generalization of Hamaker et al. [14] to get a relation between the Hilbert Transform of the acquired fan-beam data and the Hilbert transform of the reference object projections. The result is given below:

$$
\left.\frac{1}{\left|\operatorname{det} A_{\beta}\right|} p_{O H}(\boldsymbol{n}, s)\right|_{s=a_{0}(\beta) \cdot \boldsymbol{n}}=g_{H_{\beta}}\left(\beta, A_{\beta}^{\mathrm{T}} \boldsymbol{n}\right)
$$

where $p_{\mathrm{OH}}$ is the Hilbert Filtered Parallel beam Projections of a ray perpendicular to $\boldsymbol{n}$ and the distance of $s$ from the origin along $\boldsymbol{n}$, thus Hilbert transform is in the direction of $\boldsymbol{n}$ whereas $g_{H_{\beta}}$ is the Hilbert filtered fanbeam projections along the direction $A_{\beta}^{\mathrm{T}} \boldsymbol{n}$ and $\boldsymbol{a}_{0}(\beta)$ is the virtual source trajectory given by:

$\boldsymbol{a}_{0}(\beta)=\Gamma_{\beta}(\boldsymbol{a}(\beta))$.

On modification of the condition stated by Noo et al. [11] (Noo's formula will be briefed in the next section) for static case, the following admissibility condition is deduced for the dynamic CT: Region of interest (ROI) in 
a dynamic object can be successfully reconstructed if all lines passing through the neighborhood of ROI intersects the virtual trajectory $\boldsymbol{a}_{0}(\beta)[10]$.

\subsection{Fan-Beam Reconstruction Formula Based on Hilbert Filtering}

A new class of algorithm based on Hilbert FBP (HFBP) has been derived [11] from the relation given in [14] for equi-angular static fan-beam projections, which is given by

$$
\begin{aligned}
& f_{0}\left(\boldsymbol{x}_{0}\right) \\
& =\left.\frac{1}{2 \pi} \int_{0}^{2 \pi} \mathrm{d} \beta \frac{1}{\left\|\boldsymbol{x}_{0}-\boldsymbol{a}_{0}(\beta)\right\|} w(\beta, \phi) g_{F}^{m}(\beta, \phi)\right|_{\phi=\phi^{\prime}\left(\beta, x_{0}\right)}
\end{aligned}
$$

where

$$
g_{F}^{m}(\beta, \phi)=\int_{-\gamma_{m}}^{\gamma_{m}} \mathrm{~d} \gamma h_{H}(\sin (\phi-\gamma))\left(\frac{\partial}{\partial \beta}+\frac{\partial}{\partial \gamma}\right) g^{m}(\beta, \gamma),
$$

$\phi^{\prime}$ is the angle characterizing the ray that diverges from $\boldsymbol{a}_{0}(\beta)$ and contains $\boldsymbol{x}_{0}$, the projection data is characterized by $\beta$ and $\gamma$

$$
g^{m}(\beta, \gamma)=g(\beta, \alpha),
$$

$\boldsymbol{\alpha}$ and $\boldsymbol{n}$ are defined through parametrization with respect to $\gamma$ and $\phi$

$$
\boldsymbol{\alpha}=\cos \gamma \boldsymbol{e}_{u}+\sin \gamma \boldsymbol{e}_{v} \text { and } \boldsymbol{n}=-\sin \phi \boldsymbol{e}_{u}+\cos \phi \boldsymbol{e}_{v},
$$

$w\left(\beta, \phi^{\prime}\right)$ is the redundancy weight and

$$
\phi^{\prime}\left(\beta, \boldsymbol{x}_{0}\right)=\arctan \left(\frac{\boldsymbol{x}_{0} \cdot \boldsymbol{e}_{v}}{R_{0}+\boldsymbol{x}_{0} \cdot \boldsymbol{e}_{u}}\right)
$$

where $x_{0}$ is the point, to be reconstructed, on the reference object.

Two unit orthogonal vectors $\boldsymbol{e}_{u}$ and $\boldsymbol{e}_{v}$ given in the above equation are described as follows:

$\boldsymbol{e}_{v}(\beta)=\left[\begin{array}{ll}-\sin \beta & \cos \beta\end{array}\right]^{\mathrm{T}}$, which is tangent to the source trajectory at $\boldsymbol{a}_{0}(\beta)$ and

$\boldsymbol{e}_{u}(\beta)=\left[\begin{array}{ll}-\cos \beta & -\sin \beta\end{array}\right]^{\mathrm{T}}$, which is opposite to the direction of $\boldsymbol{a}_{0}(\beta)$.

The Hilbert FBP divergent beam reconstruction algorithms have spatially more uniform resolution and noise performance, and reduced divergent beam artefacts as a result of $\frac{1}{\left\|\boldsymbol{a}_{0}(\beta)-\boldsymbol{x}_{0}\right\|}$ backprojection weight instead of the $\frac{1}{\left\|\boldsymbol{a}_{0}(\beta)-\boldsymbol{x}_{0}\right\|^{2}}$ weight in ramp FBP algorithm [15].

Noise non uniformity is appeared in the reconstructed image since ramp FBP algorithm is implemented using shift invariant filter [16]. Noise uniformity is achieved with shift variant filter [17]. Recently, two fan-beam algorithms $[13,18]$ with no backprojection weight have been derived to address the position or distance dependent non-uniformity of resolution and noise variance by eliminating the backprojection weight. Extension of these two algorithms for cone-beam tomography has been given in [19].

\subsection{Fan-Beam Reconstruction Formula with No Backprojection Weight}

As discussed earlier, fan-beam FBP reconstruction formula (4) is convoyed with a position dependent weight, thus increasing the computational cost of the overall algorithm. The solution to eliminating this weight has been given in [13] by choosing a weighting function proportional to the back-projection weight

$$
w\left(\beta, \phi^{\prime}\right)=\frac{\left\|\boldsymbol{x}_{0}-\boldsymbol{a}_{0}(\beta)\right\|}{2 R_{0} \cos \phi^{\prime}}
$$

and such that

$$
w\left(\beta, \phi^{\prime}\right)+w\left(\beta_{c}, \phi_{c}^{\prime}\right)=1
$$

where $\beta_{c}$ represents a complementary source location such that $\boldsymbol{a}_{0}(\beta), \boldsymbol{x}_{0}$ and $\boldsymbol{a}_{0}\left(\beta_{c}\right)$ are collinear and $\phi_{c}^{\prime}$ is the angle characterizing the fan-beam ray emanating from $\boldsymbol{a}_{0}\left(\beta_{c}\right)$ and containing $\boldsymbol{x}$. Thus the value of radon transform is same for the rays characterized by $\left(\beta, \phi^{\prime}\right)$ and $\left(\beta_{c}, \phi_{c}^{\prime}\right)$. Therefore, by choosing the weighting function (9), the position dependent back-projection weight is eliminated, yielding the below given formula:

$$
f_{0}\left(x_{0}\right)=\frac{1}{4 \pi} \int_{0}^{2 \pi} \mathrm{d} \beta \frac{1}{R_{0} \cos \phi^{\prime}} g_{F}^{m}\left(\beta, \phi^{\prime}\right) .
$$

This is the fan-beam FBP reconstruction algorithm with no back-projection weight for equi-angular curved detector geometry.

\section{Incorporation of Motion Model in Static Fan-Beam FBP Reconstruction Formula}

In this section, we shall brief the readers about the motion compensation algorithm given in [10] to tackle motion artefacts arising due to affine deformations during the data acquisition process. This formula was derived by incorporating motion model or deformation model into the fan-beam FBP reconstruction formula given in [11]. Since the static formula is convoyed with a position dependent back-projection weight, therefore the affine deformation compensation formula for dynamic $\mathrm{CT}$ is also computationally expensive due to the back-brojection weight inhereted from formula given in [11]. We present a technique to eliminate this position dependent backprojection weight for translational, uniform scaling and rotational deformation by choosing the weighting func- 
tion similar to the one chosen in [13] for the static case.

\subsection{Reconstruction Formula with \\ Backprojection Weight: Compensation of Affine Deformation}

Let $g_{\beta}(\beta, \gamma)$ be the deformed projection data. A fanbeam ray is parametrized by the parameters $\beta$ and $\gamma$, where $\gamma$ is the angle between a ray of interest and the line connecting source position to the origin. With reference to the dynamic object, the normal of a ray, passing through a point to be reconstructed, $\boldsymbol{x}$ is denoted as $\eta(\beta, \zeta)$, where $\zeta$ is the angle of the ray connecting source and point to be reconstructed with in the fan: $\eta(\beta, \zeta)=-\sin (\zeta) \boldsymbol{e}_{u}+\cos (\zeta) \boldsymbol{e}_{v}$. For each real x-ray, diverging from the vertex point, there exist a virtual $\mathrm{x}$-ray path given by,

$$
\frac{\boldsymbol{x}_{0}-\boldsymbol{a}(\beta)}{\left\|\boldsymbol{x}_{0}-\boldsymbol{a}_{0}(\beta)\right\|}=\Gamma\left(\frac{\boldsymbol{x}-\boldsymbol{a}(\beta)}{\|\boldsymbol{x}-\boldsymbol{a}(\beta)\|}\right)
$$

where $\frac{\boldsymbol{x}-\boldsymbol{a}(\beta)}{\|\boldsymbol{x}-\boldsymbol{a}(\beta)\|}$ is the unit vector along the real x-ray path and $\frac{\boldsymbol{x}_{0}-\boldsymbol{a}_{0}(\beta)}{\left\|\boldsymbol{x}_{0}-\boldsymbol{a}_{0}(\beta)\right\|}$ is the unit vector along the virtual $x$-ray path.

Consider $\theta_{\phi}=\left[\begin{array}{ll}-\sin \phi & \cos \phi\end{array}\right]^{\mathrm{T}}$ as the normal to the virtual ray, joining the source and the point to be reconstructed on the reference object.

With Reference [10], we directly write the following relation,

$$
\eta(\beta, \zeta)=\frac{A_{\beta}^{-\mathrm{T}} \boldsymbol{\theta}_{\phi}}{\left\|A_{\beta}^{-T} \boldsymbol{\theta}_{\phi}\right\|}
$$

And the fan-beam reconstruction formula from deformed projection data is given by

$$
\begin{aligned}
& f_{0}(\boldsymbol{x}) \\
& =\left.\frac{1}{2 \pi} \int_{\Lambda_{\left(\mathrm{x}_{0}\right)}^{\Gamma}} \mathrm{d} \beta \frac{1}{\left\|\boldsymbol{a}_{0}(\beta)-\boldsymbol{x}_{0}\right\|}\left[w(\beta, \zeta) g_{F_{\beta}}^{m}(\beta, \zeta)\right]\right|_{\zeta=\zeta^{*}\left(x_{0}, \beta\right)}
\end{aligned}
$$

where

$$
\begin{aligned}
g_{F_{\beta}}^{m}(\beta, \zeta)= & \frac{\partial}{\partial \beta} g_{w H_{\beta}}^{m}(\beta, \zeta(\beta, \boldsymbol{\eta})) \\
& +\frac{\partial}{\partial \zeta} g_{w H_{\beta}}^{m}(\beta, \zeta(\beta, \boldsymbol{\eta})) \cdot \frac{\partial}{\left.\partial \beta\right|_{\phi}} \zeta
\end{aligned}
$$

where, the set $\Lambda^{\Gamma}\left(x_{0}\right)$ is defined as: $\forall \phi \in[0, \pi] \exists \beta_{*} \in \Lambda^{\Gamma}\left(x_{0}\right)$ such that, $\boldsymbol{x}_{0} \cdot \boldsymbol{\theta}_{\phi}=\boldsymbol{a}_{0}\left(\beta_{*}\right) \cdot \boldsymbol{x}_{0}$ with

$$
\frac{\partial \zeta}{\left.\partial \beta\right|_{\phi}}=1+\frac{1}{\left\|A_{\beta}^{-T} \eta(\zeta)\right\|}+\xi(\zeta)
$$

where

$$
\begin{aligned}
\xi= & {\left[\left(A_{\beta}^{\prime} \boldsymbol{e}_{v} \cdot A_{\beta}^{-\mathrm{T}} \boldsymbol{\eta}(\zeta)\right)\left(\boldsymbol{e}_{u} \cdot \boldsymbol{\eta}(\zeta)\right)\right.} \\
& \left.-\left(A_{\beta}^{\prime} \boldsymbol{e}_{u} \cdot A_{\beta}^{-\mathrm{T}} \boldsymbol{\eta}(\zeta)\right)\left(\boldsymbol{e}_{v} \cdot \boldsymbol{\eta}(\zeta)\right)\right],
\end{aligned}
$$

and

$$
\begin{gathered}
g_{w H_{\beta}}^{m}(\beta, \zeta)=\left|\operatorname{det} A_{\beta}\right||| A_{\beta}^{-\mathrm{T}} \eta(\zeta)||\left(g_{\beta}^{m} * h_{H}^{a n g}\right)(\beta, \zeta) \\
h_{H}^{a n g}(\zeta)=\left|\frac{\zeta}{\sin (\zeta)}\right| h_{H}(\zeta)
\end{gathered}
$$

where $h_{H}$ is the impulse response of Hilbert Filter.

For static fan-beam case, the virtual trajectory and the real trajectory is the same, similarly the reference object is the same as dynamic object. Thus $A_{\beta}=I_{d}$, where $I_{d}$ is the Identity matrix.

\subsection{Reconstruction Formula with No \\ Backprojection Weight: Compensation for Translational, Uniform Scaling and Rotational Deformations}

In this section, we use the weighting function (9), and eliminate the position dependent back-projection weight $1 /\left\|\boldsymbol{x}_{0}-\boldsymbol{a}_{0}(\beta)\right\|$ in the image reconstruction formula given by Equation (13). The weighting

$$
w\left(\beta, \zeta^{*}(\beta, \boldsymbol{x})\right)=\frac{\|\boldsymbol{a}(\beta)-\boldsymbol{x}\|}{2 R_{0} \cos \zeta^{*}}
$$

Equations (9), (10), the above Equation (19) is valid as it normalizes to one, the contribution from any line through $\boldsymbol{x}$ within the FOV. The back projection weight in the virtual acquisition geometry is given by:

$$
\frac{1}{\left\|\boldsymbol{a}_{0}-\boldsymbol{x}_{0}\right\|}
$$

where

$$
\begin{aligned}
\boldsymbol{a}_{0} & =\Gamma_{\beta}(\boldsymbol{a}) \text { and } \boldsymbol{x}_{0}=\Gamma_{\beta}(\boldsymbol{x}) \\
\therefore \boldsymbol{a}_{0} & =A_{\beta} \boldsymbol{a}+\boldsymbol{b}_{\beta} \text { and } \boldsymbol{x}_{0}=A_{\beta} \boldsymbol{x}+\boldsymbol{b}_{\beta} .
\end{aligned}
$$

Substituting the result obtained in Equation (21) to the back-projection weight we get,

$$
\frac{1}{\left\|A_{\beta} \boldsymbol{a}+\boldsymbol{b}_{\beta}-\left(A_{\beta} \boldsymbol{x}+\boldsymbol{b}_{\beta}\right)\right\|}=\frac{1}{\left\|A_{\beta}(\boldsymbol{a}-\boldsymbol{x})\right\|}=\frac{1}{\left\|\boldsymbol{a}_{0}-\boldsymbol{x}_{0}\right\|} .
$$

The key point to observe from Equation (22) is that the back-projection weight is independent of translational 
and rotational deformation, it only depends on scaling and shearing. The formula (13) compensates for time dependent affine deformation but is convoyed with a position dependent weight as discussed earlier. Therefore, if the object being imaged is subjected to translational or rotational deformation or both, then the term $\|\boldsymbol{a}(\beta)-\boldsymbol{x}\|$ in the weighting function (19) does not change (i.e.

$\left.\|\boldsymbol{a}(\beta)-\boldsymbol{x}\|=\left\|\boldsymbol{a}_{0}(\beta)-\boldsymbol{x}_{0}\right\|\right)$, but scaling and shearing affects the back-projection weight as suggested by Equation (21). We propose to eliminate the position dependent back-projection weight for rotational, uniform scaling and translational deformations or their combinations.

Concretely, for a translational deformation, the matrix is chosen as

$$
A_{\beta}=A_{\beta}^{(t r)}=\left[\begin{array}{ll}
1 & 0 \\
0 & 1
\end{array}\right]
$$

and the $\boldsymbol{b}_{\beta}$ can be chosen any vector since it does not effect the modification process of weighting term (22). In case of only rotational deformation, the transformation is done with a matrix given by

$$
A_{\beta}=A_{\beta}^{(R)}=\left[\begin{array}{cc}
\cos \theta & -\sin \theta \\
\sin \theta & \cos \theta
\end{array}\right] .
$$

therefore

$$
\frac{1}{\left\|A_{\beta}^{(R)}(\boldsymbol{a}-\boldsymbol{x})\right\|}=\frac{1}{\|\boldsymbol{a}-\boldsymbol{x}\|}=\frac{1}{\left\|\boldsymbol{a}_{0}-\boldsymbol{x}_{0}\right\|}
$$

So, back-projection weight does not change in case of rotational deformation. Now we shall discuss about uniform scaling wherein the back-projection weight changes. For uniform scaling deformation, $A_{\beta}=A_{\beta}^{(s c)} \beta$

$$
A_{\beta}^{(s c)}=\left[\begin{array}{cc} 
\pm \lambda & 0 \\
0 & \pm \lambda
\end{array}\right] \text {. }
$$

therefore,

$$
\frac{1}{\left\|A_{\beta}^{(s c)}(\boldsymbol{a}-\boldsymbol{x})\right\|}=\frac{1}{\lambda\|\boldsymbol{a}-\boldsymbol{x}\|}=\frac{1}{\left\|\boldsymbol{a}_{0}-\boldsymbol{x}_{0}\right\|}
$$

Thus, by employing the weighting function (19) and the Equation (26) in Equation (13) we deduce the following fan-beam FBP reconstruction formula:

$$
f_{0}\left(x_{0}\right)=\left.\frac{1}{2 \pi} \int_{\Lambda_{w}^{\Gamma}\left(x_{0}\right)} \frac{1}{2 R_{0} \lambda \cos \zeta}\left[g_{F_{\beta}}^{m}(\beta, \zeta)\right]\right|_{\zeta=\zeta^{*}\left(\vec{x}_{0}, \beta\right)} \mathrm{d} \beta
$$

where set $\Lambda_{w}^{\Gamma}\left(\boldsymbol{x}_{0}\right)$ is defined as:

$$
\forall \phi \in[0, \pi] \exists \beta_{*} \in \Lambda_{w}^{\Gamma}\left(x_{0}\right): \operatorname{range}\left(\beta_{*}\right)=(c, c+2 k \pi)
$$

where $k \in[1,2,3, \cdots]$ and $c$ is the initial value of $\beta$.

Equation (27) gives us the exact image reconstruction formula for compensating rotational and translational deformation during acquisition of equi-angular fan beam projections without any position dependent back-projection weight.

\subsection{Salient Features of Proposed Algorithm}

Since the technique involves derivative of the Hilbert filtered projection data, we have an advantage in terms of noise performance $[20,21]$. The implementation of modified formula exhibits less variance compared to the fanbeam algorithm with no backprojection weight since more implementation blocks in modified formula. It can be mentioned here that the modified formula gives better computational efficiency than the unmodified for dynamic case. However, we shall give execution time form both algorithm in the simulation section.

\section{Simulation Results}

We simulate projection of low contrast Shep Logan phantom by considering the static as well as rotational and scaled deformed object. The scan parameters which we have used for simulation have been given Table 1 . We have chosen $A_{\beta}$ or $A_{t}$ as diagonal matrix with following parameters $a_{11}(\beta)=a_{22}(\beta)$

$=0.2+0.1 \sin (\beta)$. And, $\boldsymbol{b}_{\beta}=\left[\begin{array}{ll}0 & 0.2\end{array}\right]$ is chosen as constant term with respect to time. Figure 2 shows the sinogram of original Shep Logan and deformed Shep Logan phantom. Firstly, the Shep Logan phantom is reconstructed using the fan-beam algorithm with no backprojection weight for a static object, which inturn yields an exact reconstruction of the Shep Logan Phantom (Figure 3(b)). Secondly, we reconstruct the Shep Logan Phantom from rotationally deformed data using fan-beam algorithm with no backprojection weight and our presented algorithm (Figures 3(c) and (d)). The observations completely abide with the theoretical and mathematical formulations and the Shep Logan phantom is exactly reconstructed. It is clearly observed from the Figure 3 that our proposed reconstruction algorithm removes the rotational motion artefacts.

\subsection{Computational Complexity}

The only difference between the Equations (27) and (13)

Table 1. Fan-beam imaging parameters used in simulations.

\begin{tabular}{cc}
\hline Imaging parameter & Value \\
\hline Number of pixels & $256^{2}$ \\
Object radius & 1.0 \\
Scanning radius & 2.4 \\
Source to detector distance & 4.8 \\
Number of source positions per turn & 450 \\
Number of detectors & 460
\end{tabular}



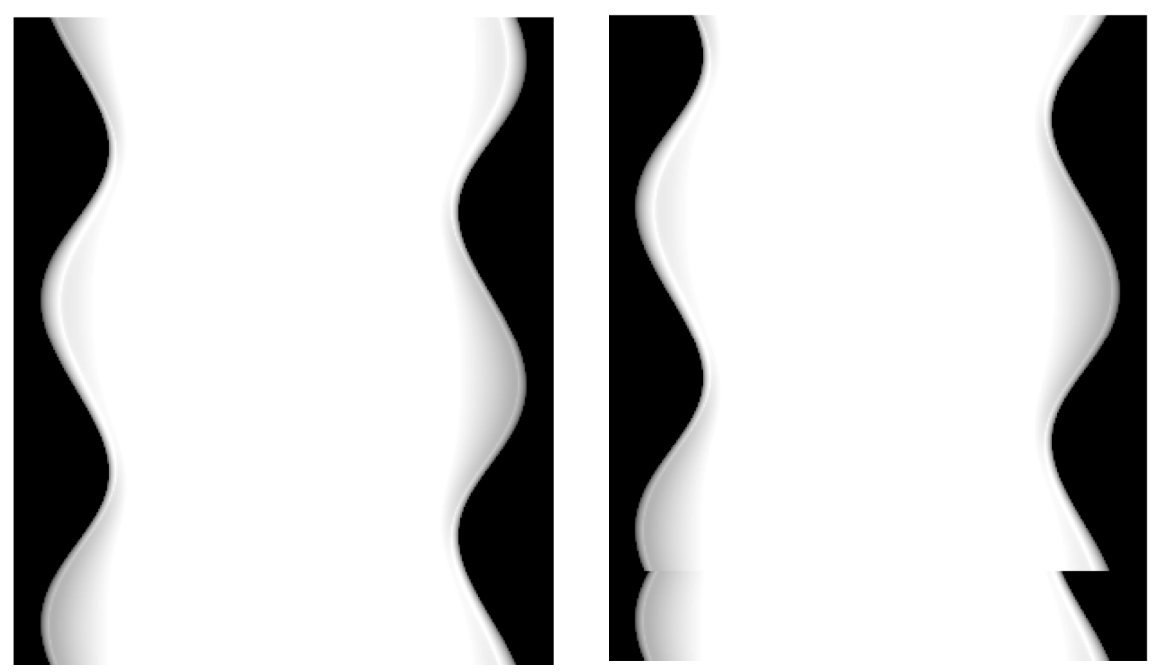

(a)

(b)

Figure 2. Sinogram: (a) Original Shep Logan phantom; (b) Deformed Shep Logan phantom.

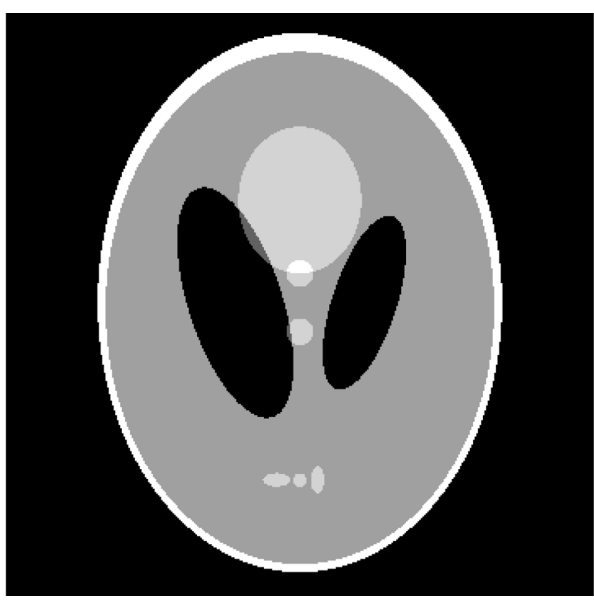

(a)

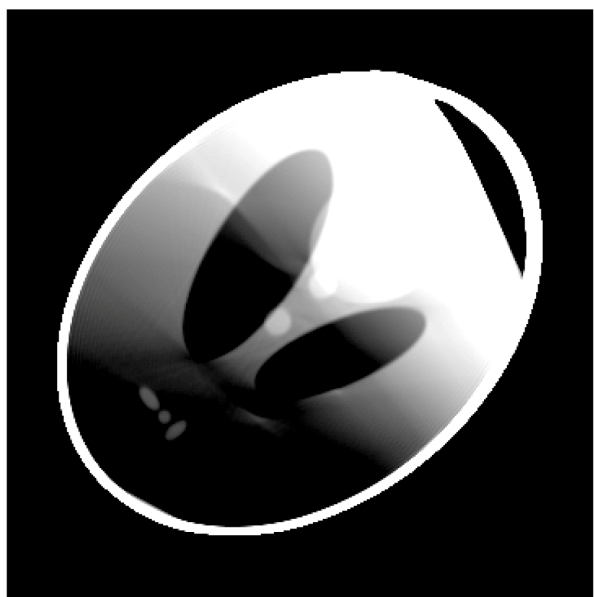

(c)

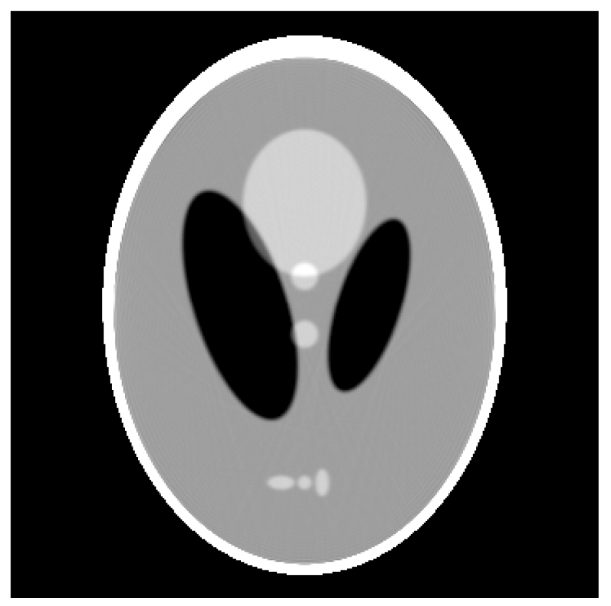

(b)

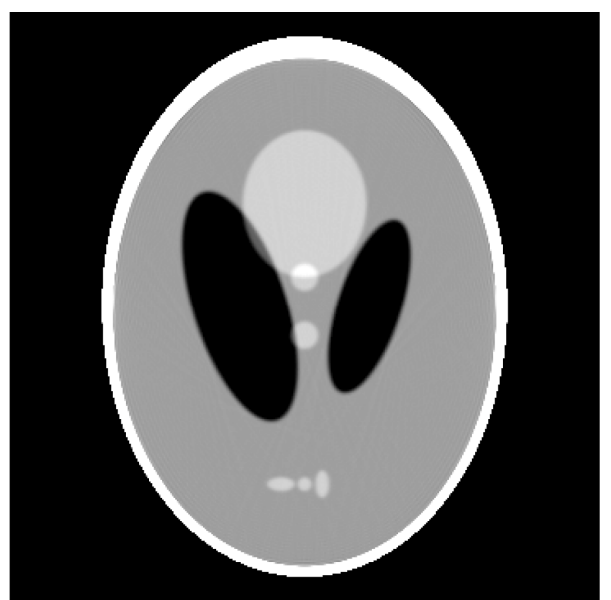

(d)

Figure 3. Reconstruction of low contrast Shepp-Logan phantom, (a) Original phantom; (b) Using fan-beam reconstruction algorithm with no backprojection weight from the static data; (c) Reconstruction with deformed data using fan-beam reconstruction algorithm with no backprojection weight; (d) Reconstruction with deformed data using modified fan-beam algorithm with no backprojection weight. $\beta(t)=30^{\circ}$ rotation is used for each source position for dynamic projections. 
Table 2. Execution time (in seconds).

\begin{tabular}{cccc}
\hline & Filtering & Backprojection & Total \\
\hline Roux et al. algorithm & 0.422 & 68.57 & 68.992 \\
Proposed algorithm & 0.46 & 20.44 & 21 \\
\hline
\end{tabular}

is the position dependent weight and redundancy weighting are replaced by the term $\frac{1}{2 R_{0} \lambda \cos \zeta}$. This term can be implemented before the backprojection. Hence, it is clearly note that the reconstruction using (27) takes less time than the reconstruction using (13). However, we have implemented proposed formula for dynamic projection using " $\mathrm{C}$ " complier. From the Table 2, it is clearly observed that computational efficiency is achieved significantly using modified fan-beam algorithm with no backprojection weight than existing exact fan-beam reconstruction for dynamic projections data.

\section{Conclusion}

In this paper, we have presented the fan-beam reconstruction algorithm with no backprojection weight for compensating time-dependent rotational deformation. Since large amount of data is needed for dynamic CT, elimination of position-dependent back-projection weight from dynamic fan-beam reconstruction algorithm given by Roux et al., facilitates more advantages in terms of computational efficiency than the same in static CT. The presented algorithm inherits the better noise and resolution characteristics same as fan-beam reconstruction algorithm with no backprojection weight for static CT. It is very clear from the above simulation results that the proposed algorithm compensates for time-dependent rotational, translational and scaling deformation and exactly reconstructs the object, under consideration without any motion artefacts.

\section{REFERENCES}

[1] M. Kachelriess and W. A. Kalender, "ElectrocardiogramCorrelated Image Reconstruction from Subsecond Spiral Computed Tomography Scans of the Heart," Medical Physics, Vol. 25, 1998, pp. 2417-2431.

http://dx.doi.org/10.1118/1.598453

[2] T. Flohr and B. Ohnesorge, "Heart Rate Adaptative Optimization of Spatial and Temporal Resolution for Electrocardiogram Gated Multislice Spiral CT of the Heart," Journal of Computer Assisted Tomography, Vol. 25, No. 6, 2001, pp. 907-923. http://dx.doi.org/10.1097/00004728-200111000-00014

[3] D. R. Gilland, B. A. Mair, J. E. Bowsher and R. J. Jaszczak, "Simultaneous Reconstruction and Motion Estimation for Gated Cardiac ECT," IEEE Transactions on Nuclear Science, Vol. 49, No. 5, 2002, pp. 2344-2349. http://dx.doi.org/10.1109/TNS.2002.803820
[4] J. De Murcia, "Reconstruction d'Images Cardiaques en Tomographie d'Emission Monophotonique a I'Aide de Modeles Spatio Temporels," PhD Thesis, Institut National Polytechnique de Grenoble, 1996.

[5] C. Blondel, R. Vaillant, G. Malandain and N. Ayache, "3D Tomographic Reconstruction of Coronary Arteries Using a Precomputed 4D Motion Field," Proceedings of 7th International Conference on Fully 3D Reconstruction in Radiology and Nuclear Medicine, Saint Malo, July 2003.

[6] S. Bonnet, A. Koenig, S. Roux, P. Hugonnard, R. Guillenaud and P. Grangeat, "Dynamic X-Ray Computed Tomography," Proceedings of the IEEE, Vol. 91, No. 10, 2003, pp. 1574-1587.

http://dx.doi.org/10.1109/JPROC.2003.817868

[7] C. J. Ritchie, C. R. Crawford, J. D. Gowvin, K. F. King and Y. Kim, "Correction of Computed Tomography Motion Artefacts Using Pixel-Specific Back-Projection," IEEE Transactions on Medical Imaging, Vol. 15, No. 3, 1996, pp. 333-342. http://dx.doi.org/10.1109/42.500142

[8] P. Grangeat, A. Koenig, T. Rodet and S. Bonnet, "Theoretical Framework for a Dynamic Cone-Beam Reconstruction Algorithm Based on a Dynamic Particle Model," Physics in Medicine and Biology, Vol. 47, No. 15, 2002, pp. 2611-2625.

http://dx.doi.org/10.1088/0031-9155/47/15/304

[9] W. Lu and T. R. Mackie, "Tomographic Motion Detection and Correction Directly in Sinogram Space," Physics in Medicine and Biology, Vol. 47, No. 8, 2002, pp. 1267 1284. http://dx.doi.org/10.1088/0031-9155/47/8/304

[10] S. Roux, L. Desbat, A. Koeing and P. Grangeat, "Exact Reconstruction in 2D Dynamic CT Compensation of TimeDependent Affine Deformations," Physics in Medicine and Biology, Vol. 49, No. 11, 2004, pp. 2169-2182. http://dx.doi.org/10.1088/0031-9155/49/11/004

[11] F. Noo, M. Defrise, R. Clackdoyle and H. Kudo, "Image Reconstruction from Fan Beam Projections on Less than a Short-Scan," Physics in Medicine and Biology, Vol. 47, No. 14, 2002, pp. 2525-2546. http://dx.doi.org/10.1088/0031-9155/47/14/311

[12] A. Katsevich, "An Improved Exact Filtered Backprojection Algorithm for Spiral Computed Tomography," Advances in Applied Mathematics, Vol. 32, No. 4, 2004, pp. 625-825.

[13] F. Dennerlein, F. Noo, J. Hornegger and G. Lauritsch, "Fan-Beam Filtered-Backprojection Reconstruction without Backprojection Weight," Physics in Medicine and Biology, Vol. 52, No. 11, 2007, pp. 3227-3240. http://dx.doi.org/10.1088/0031-9155/52/11/019

[14] C. Hamaker, K. T. Smith, D. C. Solmon and S. L. Wagner, "The Divergent Beam X-Ray Transform," Rockey Mountain Journal of Mathematics, Vol. 10, No. 1, 1980, pp. 253-283. http://dx.doi.org/10.1216/RMJ-1980-10-1-253

[15] A. C. Kak and M. Slaney, "Principles of Computerized Tomographic Imaging,” IEEE Press, New York, 1987.

[16] G. L. Zeng, "Nonuniform Noise Propagation by Using the Ramp Filter in Fan-Beam Computed Tomography," IEEE Transactions on Medical Imaging, Vol. 23, No. 6, 2004, 
pp. 690-695. http://dx.doi.org/10.1109/TMI.2004.826943

[17] X. Pan and L. Yu, "Image Reconstruction with ShiftVariant Filtration and Its Implication for Noise and Resolution Properties in Fan-Beam Computed Tomography," Medical Physics, Vol. 30, 2003, pp. 590-600. http://dx.doi.org/10.1118/1.1556608

[18] J. You and G. L. Zeng, "Hilbert Transform Based FBP Algorithm for Fan-Beam CT Full and Partial Scans," IEEE Transactions on Medical Imaging, Vol. 26, No. 2, 2007, pp. 190-199.

http://dx.doi.org/10.1109/TMI.2006.889705

[19] A. V. Narasimhadhan and K. Rajgopal, "FDK-Type Al- gorithms with no Backprojection Weight for Circular and Helical Cone-Beam CT," International Journal of Biomedical Imaging, Vol. 2012, 2012.

[20] H. Kudo, F. Noo, M. Defrise and R. Clackdoyle, "New Super Short-Scan Algorithm for Fan-Beam and ConeBeam Reconstruction," Proceedings of IEEE MIC, M5-3, 2002, pp. 902-906.

[21] A. A. Zamyatin, K. Taguchi and M. D. Silver, "Practical Hybrid Convolution Algorithm for Helical CT Reconstruction," IEEE Transactions on Nuclear Science, Vol. 53, No. 1, 2006, pp. 167-174.

http://dx.doi.org/10.1109/TNS.2005.862973 